\title{
Lighting Quality Criteria Based on the Luminance Spatial-Angular Distribution
}

\author{
Georgy Boos ${ }^{l}$, Vladimir Budak ${ }^{I}$, Tatyana Meshkova ${ }^{l}$ and Victor Zheltov ${ }^{l}$ \\ ${ }^{1}$ Light Engineering Department, National research university "MPEI”, Krasnokazarmennaya 14, Moscow, \\ 111250, Russia
}

\begin{abstract}
The article is devoted to assessing lighting quality based on lighting engineering design's spatial-angular brightness distribution (LSAD). The main problems of modern lighting design related to the modeling of scenes based on the emissivity equation and restrictions on the use, in this regard, as the main criterion for the lighting quality of Unified Glare Rating (UGR), are considered. The mathematical foundations of the use of LSAD in the practice of lighting engineering design are proposed. The integral equation LSAD is obtained, which allows modeling the brightness at an arbitrary point of the scene volume. A method for solving the formulated equation based on double local estimates of the Monte Carlo method is proposed. The formulated algorithm for calculating LSAD is view-independent: LSAD visualizes the lighting scene at all fixed points. Methods for storing the calculated LSAD are proposed. Based on the LSAD, a new criterion for lighting quality is formulated, which was experimentally tested in a full-scale experiment to evaluate the lighting of Moscow Metro stations. In the experiment, the proposed criterion and UGR were compared. One calculated the quality criteria by the station's photos, and their correlation with observers' assessment was found. Computer models of stations were created, in which quality criteria were also calculated from the experiment viewpoints - obtained good correspondence of the calculations with the estimates of observers.
\end{abstract}

\section{Keywords}

Lighting Installation, Lighting Quality, Global Illumination, Unified Glare Rating

\section{Introduction}

Modern three-dimensional computer graphics have reached truly phenomenal heights. Programs for visualizing three-dimensional scenes allow us to obtain truly photo-realistic images, which became possible due to the rapid development of computer technology and methods for solving the global illumination equation [8]. One of the practice areas that the development of computer graphics has influenced is lighting engineering. The key task in lighting engineering is the design of lighting installations, which is to find the number, types, and arrangement of lamps. Two decades ago, this was an extremely time-consuming routine engineering calculation. Today at the heart of this process, as in computer graphics, is modelling the solution of the global illumination equation (GIE). However, if a key task in computer graphics is to obtain a "realistic" image, then there is a task of correct calculation of illumination and luminance in lighting engineering. While the designer is guided by normative documents, defining qualitative and quantitative indicators of coverage.

In modern regulatory documents for non-special lighting installations (office, industrial, commercial, etc.), illumination and various parameters derived from it (the ratio of minimum illumination to maximum, etc.) are normalized as a quantitative characteristic. As a rule, all calculations are conducted for illumination on the floor of the room, or an imaginary working plane located at the

GraphiCon 2021: 31st International Conference on Computer Graphics and Vision, September 27-30, 2021, Nizhny Novgorod, Russia EMAIL: BoosGeorV@mpei.ru (G. Boos); budakvp@gmail.com (V. Budak); zheltov@list.ru (V.Zheltov), tvmesh@mail.ru (T. Meshkova) ORCID: 0000-0001-9725-4266 (G. Boos); 0000-0003-4750-0160 (V. Budak); 0000-0001-6768-2518 (V. Zheltov); 0000-0003-1008-2161 (T. Meshkova) 
table's height. However, the illumination is an integral characteristic of the incident light, while the human eye reacts to light reflected from the surface. That is, if you take a completely black surface with a reflection coefficient of zero, then formally, you can get the required illumination on it. At the same time, visually, we will not see anything since the light will not be reflected from the surface. From the point of view of the human visual organ, it is necessary to normalize the luminance. The current situation is quite understandable since the calculation and measurement of luminance were complicated until recently.

This problem is partially eliminated by the introduction of a unified glare rating (UGR) among a variety of qualitative indicators for non-special installations

$$
U G R=8 \lg \left[\frac{0,25}{L_{a}} \sum_{i=1}^{N} \frac{L_{i}^{2} \omega_{i}}{p_{i}^{2}}\right],
$$

where $L_{i}$ is the luminance of the glitter source, $\mathrm{cd} / \mathrm{m}^{2} ; \omega_{i}$ is the angular size of the glitter source; $p_{i}$ is the light source position index relative to the sightline; $L_{a}$ is the adaptation luminance, $\mathrm{cd} / \mathrm{m}^{2}$.

Accordingly, the UGR allows expressing the lighting quality in just one figure, making it possible to include it in regulatory documents. Today, when designing non-special LI, the designer is guided by the illumination as a quantitative characteristic and UGR as a qualitative assessment of lighting. UGR answers the question-how comfortable a person will be within the lighting system.

However, the formula (1) is valid only for small-angle uniform glare sources, i.e., it cannot consider extended non-uniform glare. Moreover, the DIALux and Relux programs widely used in lighting design are based on the finite element method for the radiosity equation in the diffuse approximation and not on the global illumination equation for luminance. Therefore, it is obvious that secondary glare cannot be considered in principle. The actual UGR only includes the outlet holes of the luminaires themselves directly. In lighting systems with hidden lighting fixtures, the quality of UGR-based lighting cannot be calculated.

However, as shown later in the paper, with a certain approach to UGR, such calculations are possible when using luminance images. In this case, based on contrasts, sources of glare can be found, which can be considered individual pixels that contribute to the UGR. Including in the case of sources' hidden location, their glare can be distinguished based on contrasts, which was done for the experiment described later in the article. Moreover, it is the contrast that forms the basis of the new quality criterion we are considering.

A significant step forward in lighting design is DIALux Evo's introduction based on the photon map method. However, it still uses the diffuse approximation and, accordingly, the UGR calculation method has not changed.

Thus, LI is designed by assessing approximately how comfortable a person will be in it and normalize the invisible characteristic-illumination. However, at the beginning of the last century, it was suggested that the luminance spatial-angular distribution (LSAD) plays a key role in the issue of comfort [6]. Note that the lighting quality depends on three main luminance distributions: by space, by spectrum, and by time, and all of them have some relationship. Here and further in work, when we talk about lighting quality only by the distribution over space.

\section{Luminance spatial-angular distribution}

Modelling of lighting installations is based on the equation of global illumination, well known in computer graphics, first obtained [8]:

$$
L(\mathbf{r}, \hat{\mathbf{l}})=L_{0}(\mathbf{r}, \hat{\mathbf{l}})+\frac{1}{\pi} \int L\left(\mathbf{r}, \hat{\mathbf{l}}^{\prime}\right) \sigma\left(\mathbf{r} ; \hat{\mathbf{l}}, \hat{\mathbf{l}}^{\prime}\right)\left|\left(\hat{\mathbf{N}}, \hat{\mathbf{l}}^{\prime}\right)\right| \hat{\mathbf{l}}^{\prime},
$$

where $L(\mathbf{r}, \hat{\mathbf{l}})$ is the luminance at a point $r$ in the direction $\hat{\mathbf{l}}, \sigma\left(\mathbf{r} ; \hat{\mathbf{i}}, \hat{\mathbf{l}}^{\prime}\right)$ is bidirectional scattering (reflectance or transmittance) distribution function, $L_{0}$ is the source luminance, $\hat{\mathbf{N}}$ is the normal to a scene surface element at a point $\mathbf{r}$.

The integral equation in the form (2) is of little use for its solution by the Monte Carlo method. In the Monte Carlo method, the central place is occupied by statistical modeling of trajectories, which is 
usually built on the equation's core. Modeling trajectories in space is obvious but building a trajectory on a three-dimensional surface is extremely difficult. We transform the integral in equation (2) to an integral over three-dimensional space. To do this, we select the origin of the ray exiting from $r$ in the direction of $\hat{\mathbf{l}}$. Using the properties of the $\delta$-function, we add the integral over the ray as the radius vector from the reference point. After simple transformations, equation (2) is transformed into the expression:

$$
L(\mathbf{r}, \hat{\mathbf{l}})=L_{0}(\mathbf{r}, \hat{\mathbf{l}})+\frac{1}{\pi} \int L\left(\mathbf{r}^{\prime}, \hat{\mathbf{l}}^{\prime}\right) \sigma\left(\mathbf{r} ; \hat{\mathbf{l}}^{\prime}, \hat{\mathbf{l}}\right) G\left(\mathbf{r}^{\prime}, \mathbf{r}\right) d^{3} r^{\prime}
$$

where

$$
G\left(\mathbf{r}^{\prime}, \mathbf{r}\right)=\left.\frac{\left.\mid \hat{\mathbf{N}}(\mathbf{r}), \mathbf{r}-\mathbf{r}^{\prime}\right) \mid}{\left(\mathbf{r}-\mathbf{r}^{\prime}\right)^{3}} \Theta\left(\mathbf{r}^{\prime}, \mathbf{r}\right)\left|\frac{d \Pi\left(\mathbf{r}-\xi \hat{\mathbf{I}}^{\prime}\right)}{d \xi}\right|\right|_{\xi=\mathbf{r}-\mathbf{r}_{0} \mid} \delta\left(\Pi\left(\mathbf{r}-\left|\mathbf{r}-\mathbf{r}^{\prime}\right| \hat{\mathbf{i}}^{\prime}\right)\right)
$$

$\Pi\left(\mathbf{r}-\left|\mathbf{r}-\mathbf{r}_{0}\right| \hat{\mathbf{l}}^{\prime}\right)=0$ is the equation describing the scene surfaces, $\hat{\mathbf{l}}^{\prime}=\mathbf{r}-\mathbf{r}_{0} /\left|\mathbf{r}-\mathbf{r}_{0}\right|, \Theta$ is a function that tracks shading.

Equation (3) is written for a point located on the surface П. However, the definition of the quality of lighting is related to the observer, that is, the point located in the volume of scene $V$.

Consider an equation concerning an arbitrary point in space. Let us define the luminance distribution $L(\mathbf{r}, \hat{\mathbf{l}})$ on some closed surface $\Sigma$ defined by the equation $\Pi(\mathbf{r})=0$. We need to determine the luminance distribution $L_{V}(\mathbf{r}, \hat{\mathbf{l}})$ at an arbitrary point $\mathbf{r}$ of volume $V$ bounded by the surface $\Sigma$. The volume is filled with a completely transparent medium. Following the radiative transfer equation's solution for a transparent medium, the luminance along the beam does not change. Therefore, the luminance at the point $\mathbf{r}$ in the direction $\hat{I}$ will be equal to the luminance of the surface at the point of intersection of the surface with the ray from the point $\mathbf{r}$ in the direction $\hat{\mathbf{l}}$

$$
L_{V}(\mathbf{r}, \hat{\mathbf{l}})=L(\mathbf{r}-\xi \hat{\mathbf{l}}, \hat{\mathbf{l}}),
$$

where $\xi$ is the root of the ray - surface intersection equation $\Pi(\mathbf{r}-\xi \hat{\mathbf{l}})=0$.

One can give the latter relations a more convenient analytical form based on the use of the properties of the $\delta$-function

$$
L_{V}(\mathbf{r}, \hat{\mathbf{l}})=C_{01} \int_{(V)} L\left(\mathbf{r}^{\prime}, \hat{\mathbf{l}}\right) \delta\left(\Pi\left(\mathbf{r}-\left|\mathbf{r}-\mathbf{r}^{\prime}\right| \hat{\mathbf{l}}\right)\right) \delta\left(\hat{\mathbf{l}}-\frac{\mathbf{r}-\mathbf{r}^{\prime}}{\left|\mathbf{r}-\mathbf{r}^{\prime}\right|}\right) \frac{d^{3} r^{\prime}}{\left(\mathbf{r}-\mathbf{r}^{\prime}\right)^{2}},
$$

where $C_{01}=\left|\frac{d \Pi(\mathbf{r}-\xi \hat{\mathbf{l}})}{d \xi}\right|_{\xi=\left|\mathbf{r}-\mathbf{r}_{0}\right|}$ It is related to the integral of the $\delta$-function with a complex argument, and $\delta\left(\hat{\mathbf{i}}-\frac{\mathbf{r}-\mathbf{r}^{\prime}}{\left|\mathbf{r}-\mathbf{r}^{\prime}\right|}\right)$ fixes the sighting direction of luminance.

Then, by combining the last expression with the global illumination equation (3), one can finally obtain the equation for a point in the scene volume [2].

$$
L_{V}(\mathbf{r}, \hat{\mathbf{l}})=L_{0}\left(\mathbf{r}_{\Sigma}, \hat{\mathbf{l}}\right)+\frac{1}{\pi} C_{01} \int L\left(\mathbf{r}_{1}, \hat{\mathbf{l}}^{\prime}\right) \sigma\left(\mathbf{r}_{\Sigma} ; \hat{\mathbf{l}}^{\prime}, \hat{\mathbf{l}}\right) G\left(\mathbf{r}_{1}, \mathbf{r}_{\Sigma}\right) \delta\left(\Pi\left(\mathbf{r}-\left|\mathbf{r}-\mathbf{r}_{\Sigma}\right| \hat{\mathbf{l}}\right)\right) \delta\left(\hat{\mathbf{i}}-\frac{\mathbf{r}-\mathbf{r}^{\prime}}{\left|\mathbf{r}-\mathbf{r}^{\prime}\right|}\right) d^{3} r_{1} \frac{d^{3} r_{\Sigma}}{\left(\mathbf{r}-\mathbf{r}_{\Sigma}\right)^{2}},
$$

where the point $\mathbf{r}_{\Sigma}$ is the intersection point the surface $\Sigma$ with the ray from the point $\mathbf{r}$ in the direction of $\hat{1}$.

Equation (7) describes the LSAD at each point in the scene space. This allows us to assess the quality of lighting not based on evaluating individual highlights as in UGR but based on analysing the continuous LSAD.

The resulting equation can be decomposed into a Neumann series and solved by local Monte Carlo estimators [2], first proposed in atomic physics [9].

We present the solution (7) as a Neumann series, and after some transformations, we get 


$$
\begin{gathered}
L(\mathbf{r}, \hat{\mathbf{l}})=L_{0}(\mathbf{r}, \hat{\mathbf{l}})+\frac{1}{N} \sum_{i=1}^{N}\left(\frac{1}{\pi} \frac{L_{0}\left(\mathbf{r}_{1 i}, \hat{\mathbf{l}}_{1 i}\right)}{p_{1}\left(\mathbf{r}_{1 i}, \hat{\mathbf{l}}_{1 i}\right)} \frac{\sigma\left(\mathbf{r} ; \hat{\mathbf{l}}_{1 i}, \hat{\mathbf{l}}\right) G\left(\mathbf{r}_{1}, \mathbf{r}\right)}{p_{2}\left(\mathbf{r}_{1 i}, \hat{\mathbf{l}}_{1 i} \rightarrow \mathbf{r}, \hat{\mathbf{l}}\right)}+\right. \\
\left.+\frac{1}{\pi^{2}} \frac{L_{0}\left(\mathbf{r}_{1 i}, \hat{\mathbf{l}}_{1 i}\right)}{p_{1}\left(\mathbf{r}_{1 i}, \hat{\mathbf{l}}_{1 i}\right)} \frac{\sigma\left(\mathbf{r}_{2 i} ; \hat{\mathbf{l}}_{1 i}, \hat{\mathbf{l}}_{2 i}\right) G\left(\mathbf{r}_{1 i}, \mathbf{r}_{2 i}\right)}{p_{2}\left(\mathbf{r}_{1 i}, \hat{\mathbf{l}}_{1 i} \rightarrow \mathbf{r}_{2 i}, \hat{\mathbf{l}}_{2 i}\right)} \frac{\sigma\left(\mathbf{r} ; \hat{\mathbf{l}}_{2 i}, \hat{\mathbf{l}}\right) G\left(\mathbf{r}_{2 i}, \mathbf{r}\right)}{p_{2}\left(\mathbf{r}_{2 i}, \hat{\mathbf{l}}_{2 i} \rightarrow \mathbf{r}, \hat{\mathbf{l}}\right)}+\ldots\right),
\end{gathered}
$$

that can be interpreted as a Markov chain with a transition probability determined by the kernel of the equation:

$$
k\left(x_{i} \rightarrow x\right)=\frac{\sigma\left(\mathbf{r} ; \hat{\mathbf{i}}_{i}, \hat{\mathbf{i}}\right) G\left(\mathbf{r}_{i}, \mathbf{r}\right)}{p_{2}\left(x_{i} \rightarrow x\right)}
$$

Building a Markov chain allows estimating the luminance at a given point in a given sighting direction on the scene surface. In the theory of the Monte Carlo methods, such an estimate is usually called a local estimate.

It is not possible to construct a local estimation for the LSAD equation since it contains two additional $\delta$-functions: $\delta\left(\Pi\left(\mathbf{r}-\left|\mathbf{r}-\mathbf{r}_{\Sigma}\right| \hat{\mathbf{I}}\right)\right)$ and $\delta\left(\hat{\mathbf{i}}-\frac{\mathbf{r}-\mathbf{r}^{\prime}}{\left|\mathbf{r}-\mathbf{r}^{\prime}\right|}\right)$, which depend on the desired direction $\hat{\mathbf{l}}$ . In practice, this will mean that it is impossible to get into the desired direction when evaluating the kernel of the equation. To solve this problem, we need another additional node that fixes the intermediate point $\mathbf{r}_{\Sigma}$ of equation (7). This approach is called a double local estimation.

In contrast to direct modelling methods based on ray or photon tracing and counting, local estimates allow us to estimate the luminance at a given point in a given direction based on the probability of departure from the Markov chain walk path to the point under study described by the kernel of the equation [3].

Note that for the first time such a method in the phenomenological approach was formulated in the work [10].

The development of methods for solving the global illumination equation and the emergence of new methods for measuring luminance [5] allows lighting engineering to move away from the diffuse lighting model and find the luminance characteristic saw by the human eye. So, we can raise the question of a new stage in developing qualitative characteristics based on the LSAD analysis.

\section{Lighting quality based on LSAD}

To date, when designing non-special lighting installations, such as office premises, public places, shops, shopping centres, etc., in fact, one single criterion describing the quality of the installation is used - UGR. In terms of the LSAD, visibility is affected as the absolute value of the observed luminance and the ratio of the source-background luminance difference to the background luminance (adaptation luminance) - contrast [13]. The ratio of contrast to threshold contrast can serve as a criterion for lighting quality.

In the case of a continuous LSAD over the lighting scene, the natural generalization of contrast is the ratio of the gradient of the luminance distribution over the observation field to the average luminance over the field [4]. As the gradient value increases, the boundary between the light source and the background will become more defined, and the lighting quality will decrease accordingly. The larger the source and the higher the luminance gradient around the bright source, the greater the contribution to the discomfort that source makes. Note that extended glare is both a source of discomfort in real life and contributes to the adaptation luminance. The generalized contrast at a point in the scene can be determined by:

$$
K(x, y)=\frac{|\operatorname{grad}(L(x, y) p(x, y))|}{\bar{L}}
$$

where

$$
\bar{L}=\frac{1}{A} \int_{(A)} L(x, y) p(x, y) d x d y, A=\int_{(A)} d x d y
$$


$x, y$ are the point coordinates on the scene projection, $L$ is the luminance at this point in the direction of observation, $\bar{L}$ is the average over the field of view luminance, $p(x, y)$ is the weight function that takes into account the different contribution to the eye response of points located in the centre of the visual field and on the periphery. In the criterion formula, $p$ carries the same physical meaning as the position index in the UGR formula.

Therefore, it is possible to formulate the criterion for the lighting quality $Q$ as a weighted average contrast $K(x, y)$, estimated by a certain threshold:

$$
Q=\frac{1}{K_{t h}} \int K(x, y) d x d y,
$$

where $K_{t h}$ is the value of threshold contrast. One finds the value of threshold contrast from the visual task conditions.

One can see from the expression that even a slight change in luminance can lead to a change in the luminance gradient, contributing to the quality criterion in the proposed form. Simultaneously, it is obvious that changes in contrast in luminance below a certain limit will not make a real contribution. For example, it is obvious that if there is a direct light source in the field of view or glare from it in the room, contrasts in a dark corner are likely to play a role in the feeling of lighting quality. But if one formally conducts the calculation, then they will also give a contribution. Thus, contrasts below some threshold $L_{t h}$ should not actually be considered.

In [1] conducted huge research work on setting up threshold contrasts in solving the detection problem. In this work, the relationship between threshold contrast and adaptation luminance at different target angular sizes was experimentally proved.

Following this work, it is possible to define $L_{t h}$ as a certain number of threshold values. Primarily, one needs to set the minimum element size to be detected in a given visual task. For example, it can be the size of a character when reading text or a character from a certain distance. Considering the adaptation luminance, for which one can take the average luminance over the field of view, it is possible to find from Blackwell's study the threshold changes in luminance $\Delta L$ for solving this problem. For identification tasks, it is necessary to set a certain number of threshold exceedances, which allows determining the threshold luminance:

$$
L_{t h}=N \Delta L
$$

where $N$ is a number that depends on the difference between the actual problem being solved and the threshold problem.

Therefore, we take a certain number of thresholds as the threshold luminance for cutting off contrasts that do not affect the quality criterion. Then the expression for contrast can be written as

$$
K(x, y)= \begin{cases}0, & L(x, y) \leq L_{t h} ; \\ K(x, y), L(x, y)>L_{t h} .\end{cases}
$$

\section{Experimental study of a new criterion for lighting quality and UGR}

An experiment to study the proposed criterion for lighting quality was conducted in the Moscow metro [4]. One of the real visual tasks solved in the metro is reading signs with signs. It was this task that formed the basis of the experiment. Simultaneously, in lighting practice, an expert comparison of various lighting installations is often carried out among themselves, as a rule, having the same functional lighting. So, the lighting of one metro station can be compared with the lighting of another based on expert assessments - "better-worse." In this experiment, such a scale of relations was built, as the only one available in practice. Thus, the observers' task was to find on a 10-point scale how comfortable it is to perform the specified visual task when reading the sign with navigation signs. The station was photographed from the same angles with a Nikon D3100 digital camera in RAW format, and the absolute luminance values were measured with a Konika Minolta LS-100 luminance meter for subsequent normalization of photos.

The proposed quality criterion (12) was calculated from the obtained photos. A linear correlation coefficient was used to assess the relationship between the observers ' estimates and the quality criterion. 
The required number exceeding the threshold $N$ is unknown for performing the visual task in a real scene with the Moscow metro's existing lighting system and requires further clarification based on experimental data. For this purpose, the Department of Lighting Engineering plans a three-stage experiment to determine the $K_{t h}$ when performing a visual task in office and public spaces based on a standardized C-test [11], the NASA Task Load Index questionnaire (NASA-TLX) [7] and a multidimensional questionnaire developed by the Expert Forum on Indoor Lighting (EFI) [12], which should result in a table of $K_{t h}$ values for different levels of visual tasks.

At this stage, according to the absence of dependence of threshold contrasts on the background luminance in the Weber-Fechner law, we can assume that the required number of exceeding the thresholds will lie in the range from 30 to 70 . Empirically, by selecting $N$, the highest value of the correlation coefficient 0.61 was obtained, which corresponds to a noticeable correlation on the Chaddock scale, with the number of thresholds $N$ equal to 50 . In the future, it is planned to test the obtained value in the laboratory as part of the experimental work on determining the $K_{t h}$.

Figure 1 shows the scattering map of the observers' assessment and the proposed lighting quality criterion.

The question of constructing a scale of sensations of observers is not trivial. In this experiment, there was no possibility to adjust any parameters at metro stations. The time of the experiment was also extremely strictly limited. Therefore, the "order scale" was chosen. To test the selected scale's stability, the observers ' responses randomly varied within 0.25 of the nominal value. Simultaneously, the correlation coefficient was calculated for 10,000 different variations, and its deviation was no more than $5 \%$.

The question of finding the threshold luminance and finding the number of Blackwell thresholds is our next task, as well as finding the threshold contrast in equation (6). In the first calculations, the number 50 was chosen as approximately $1 / 3$ of the average luminance for stations. Figure 2 shows the correlation coefficient's dependence on the number of thresholds used in the calculation of the criterion. A noticeable correlation between the observers ' assessment and the proposed quality criterion is in the region of tens of Blackwell thresholds.

The combined index is calculated using the formula (1), which includes uniform light sources with luminance $L_{i}$, solid angle $\omega_{i}$ and position index $p_{i}$, given by a table. Simultaneously, it is not formally specified what distance should be between the sources, which suggests that each pixel of the luminance image obtained either when calculating the LSAD or when shooting with a luminometer can be considered an independent source of glare. That is, in the original luminance photo, you need to select pixels that will be close sources, as shown in Figure 3 on the example of a Moscow metro station. Then, at a known solid angle of one pixel of the image, each can be assigned its own position index.

Thus, the UGR was calculated for all metro stations participating in the experiment. The correlation coefficient UGR from the proposed lighting quality criterion was 0.73 , and UGR from the observers ' assessment was 0.62 , which is a noticeable correlation in both cases. Figure 4 shows the scattering map of the proposed UGR vs. lighting quality criterion.

Currently, the development of lighting technology is largely associated with the BIM technology (Building Information Model), based on the digital description of buildings and the equipment installed in them. This model is a method of network planning, execution, operation, and implementation of construction projects. One of the main conditions for using BIM is the collection of all the data necessary for a building project. In this case, the building project, once created, is then updated to the stage of its full implementation. Nobody can avoid design errors, but here it can be detected at an early stage of design. A single database makes the history of changes and corrections simple and understandable for all participants.

The description of lighting equipment is based on 3D models of luminaires with their photometric data. The calculation of lighting in the premises of buildings is carried out, considering the number and location of lamps in them. In the future, the association of customers, building designers, and equipment manufacturers on a single Internet platform. For most structures, the lighting of the main rooms is determined by standard schemes. Therefore, in principle, the platform can choose the database's equipment when determining the lighting quality criterion. 


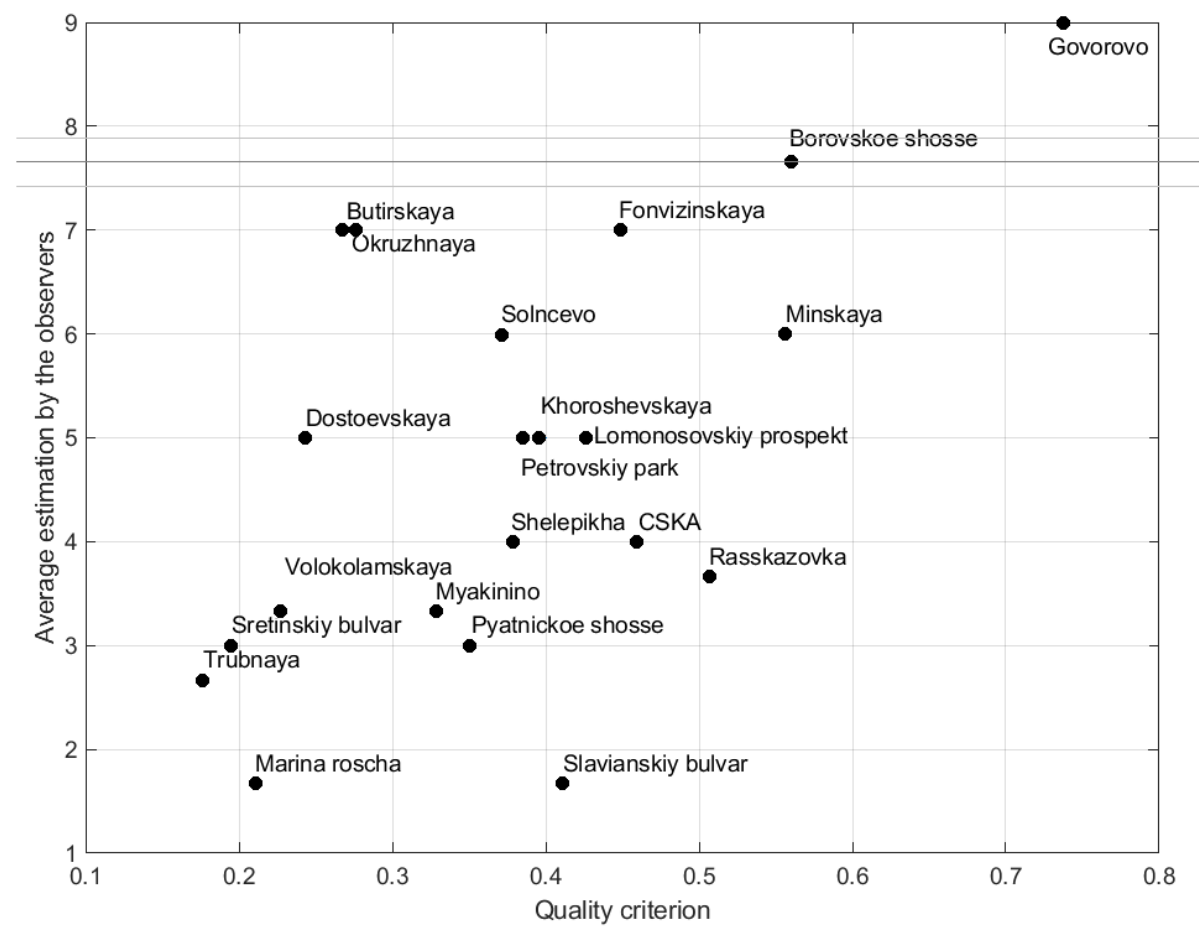

Figure 1: Scattering map of observer estimates and the proposed lighting quality criterion

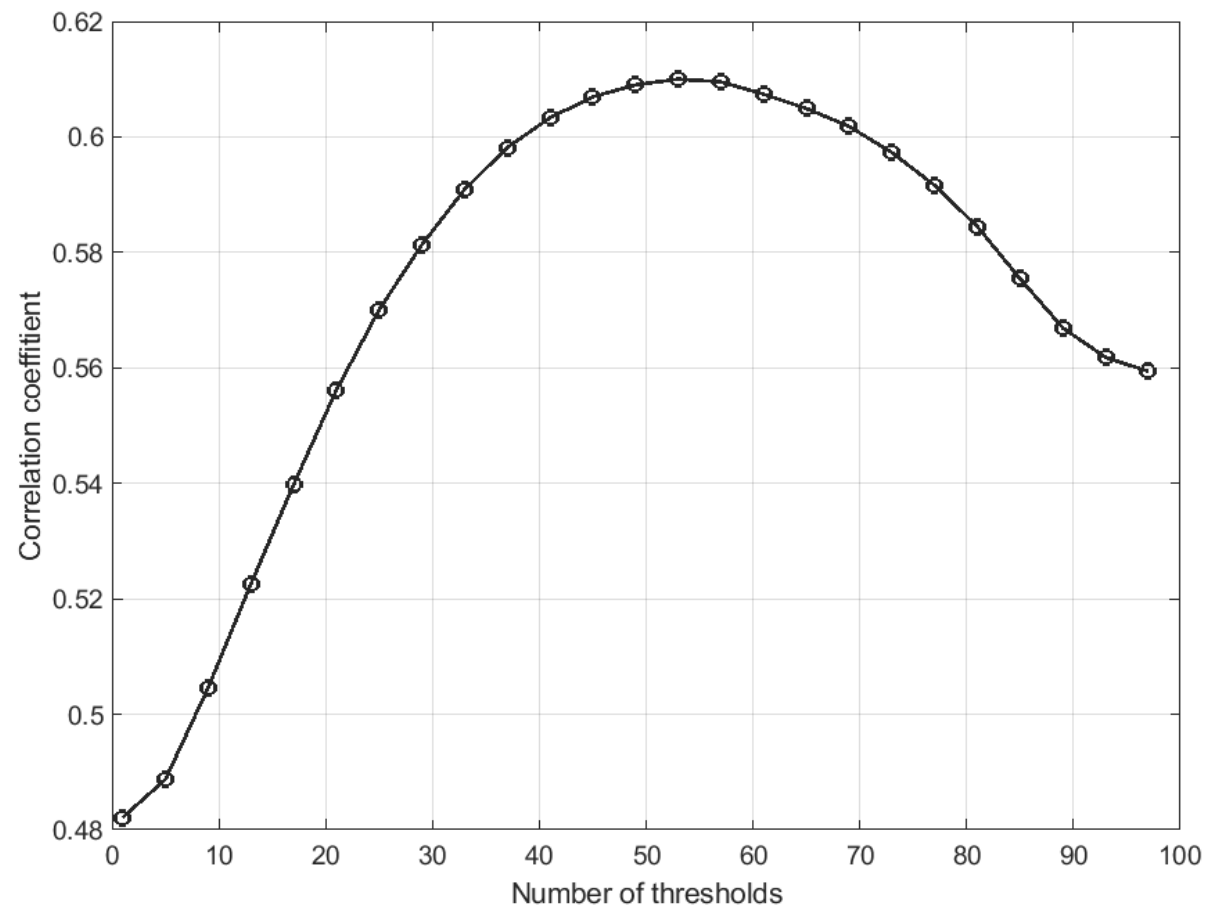

Figure 2: Correlation coefficient dependence on the number of thresholds

Currently, the development of lighting technology is largely associated with the BIM technology (Building Information Model), based on the digital description of buildings and the equipment installed in them. This model is a method of network planning, execution, operation, and implementation of construction projects. One of the main conditions for using BIM is the collection of all the data necessary for a building project. In this case, the building project, once created, is then updated to the stage of its full implementation. Nobody can avoid design errors, but here it can be detected at an early stage of design. A single database makes the history of changes and corrections simple and understandable for all participants. 

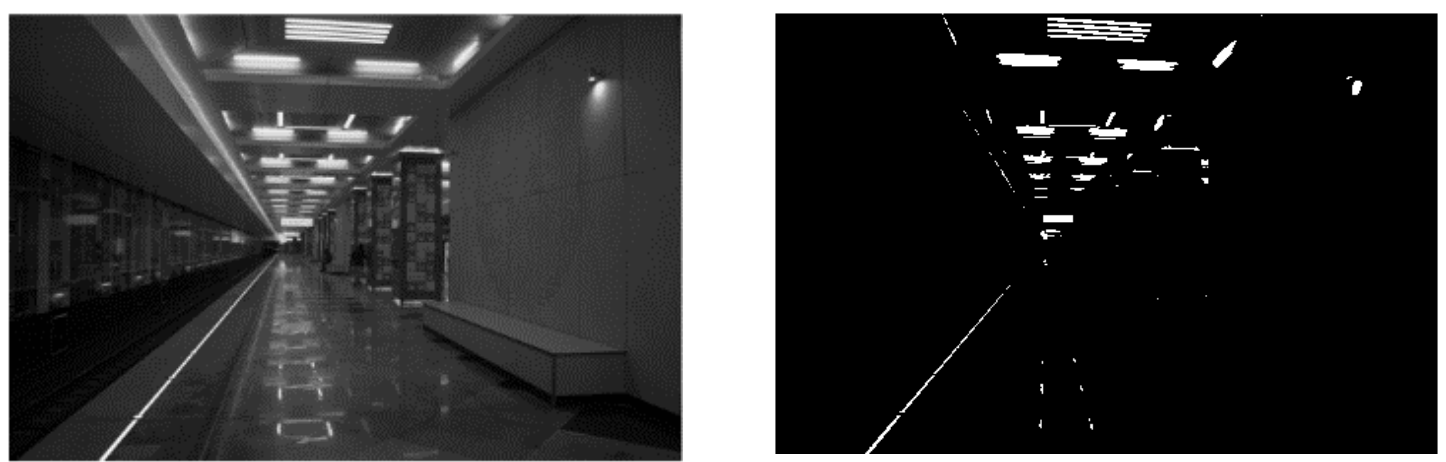

Figure 3: The definition of the pixel sources of glare

The description of lighting equipment is based on 3D models of luminaires with their photometric data. The calculation of lighting in the premises of buildings is carried out, considering the number and location of lamps in them. In the future, the association of customers, building designers, and equipment manufacturers on a single Internet platform. For most structures, the lighting of the main rooms is determined by standard schemes. Therefore, in principle, the platform can choose the database's equipment when determining the lighting quality criterion.

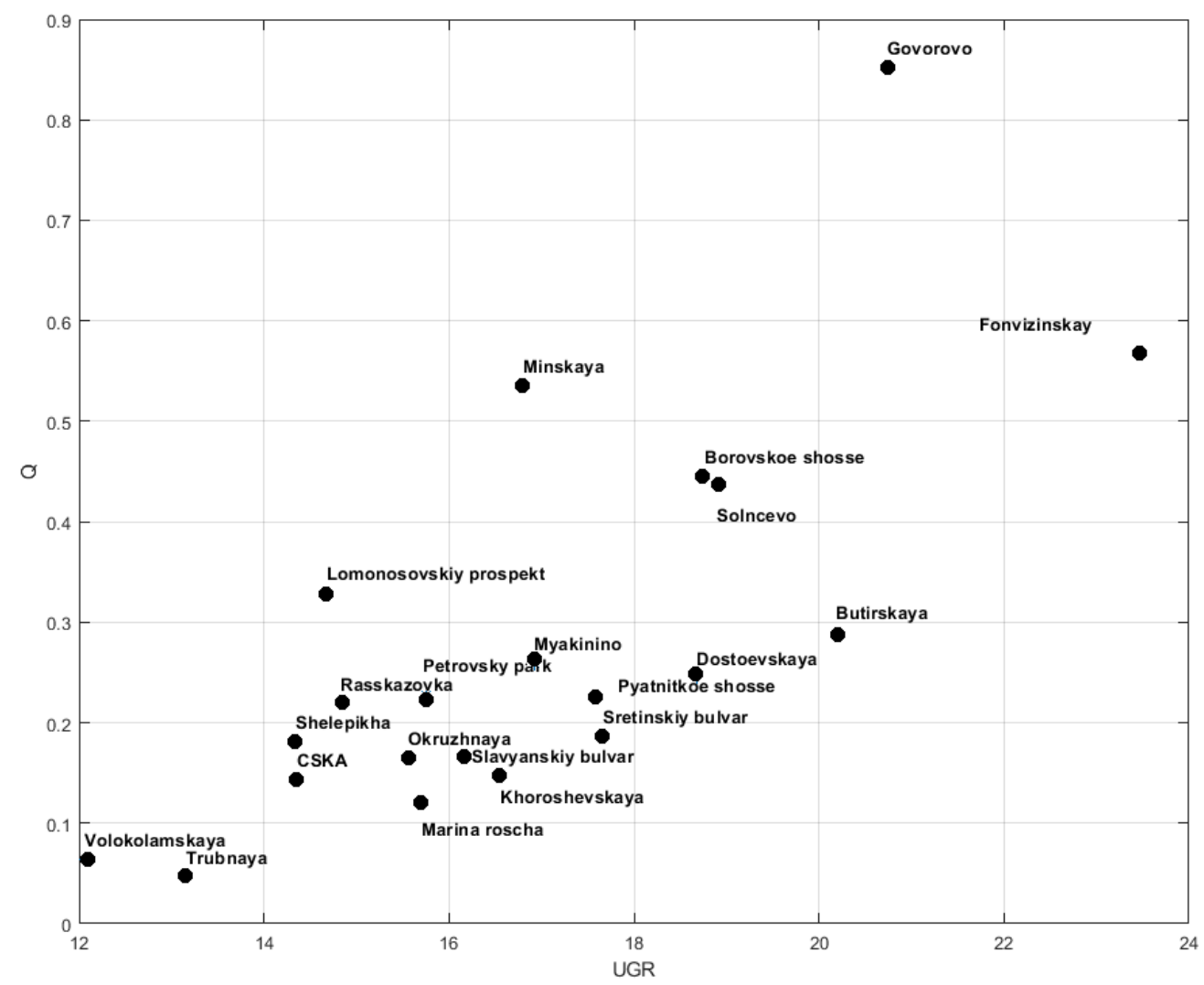

Figure 4: Scattering map of the proposed lighting quality criterion from UGR

The lighting quality criterion proposed in this paper can be the basis for optimizing the choice of equipment. There are a finite number of combinations from which the best option is selected. Today, along with direct search, some algorithms use some heuristic rules (for example, the genetic algorithm), which significantly accelerate convergence. 


\section{Conclusion}

The revolution in computer graphics in the modeling of three-dimensional scenes is also reflected in lighting technology. The formulated method of double local estimates introduces the possibility of calculating the luminance spatial-angular distribution at an arbitrary point in the volume into the lighting engineering design of lighting installations. It is a true revolution of design based on assessing the integral characteristic of luminance - illumination at specified points on the surfaces of the scene. This path in lighting engineering took a practical century from the formulation in the Ferree work that the quality of lighting depends on the luminance distribution in the field of view to the actual possibility in lighting engineering to carry out such calculations.

The paper shows the possibility of using the new opportunities that have opened for practical application, particularly for calculating the generally accepted criterion of lighting quality - UGR. Moreover, the possibility of creating new quality criteria is shown based on the use of the LSAD.

The presence of various quality criteria will allow you to move on to solving the inverse problemdesigning for a given quality. At the first stage, of course, iterating from a set of solutions with minimizing the criteria functions. This will allow lighting engineering to find its place in BIM design.

\section{References}

[1] H.R. Blackwell, Contrast Thresholds of the Human Eye, Journal of the Optical Society of America 36(11) (1946) 624-643.

[2] V. Budak, et al, Relation of instant radiosity method with local estimations of Monte Carlo method. Journal of WSCG- 24th Conference on Computer Graphics, Visualization and Computer Vision. Plzen, Czech Republic, 2016, pp.189-196.

[3] V.P. Budak, et al, Evaluation of illumination quality based on spatial-angular luminance distribution, Light \& Engineering 25(4) (2017) 24-31.

[4] V.P. Budak, et al, Experimental study of the new criterion of lighting quality based on analysis of luminance distribution at Moscow metro stations, Light \& Engineering, 28(3) (2020) 98-105.

[5] H. Cai, Luminance gradient for evaluating lighting, Lighting Research \& Technology, 48(2) (2013) 155-175.

[6] C. Ferree, et al, The efficiency of the eye under different conditions of lighting, Trans. Illum. Eng. Soc. 10 (1915) 407-447.

[7] S.G. Hart, L.E. Staveland, Development of the NASA-TLX (Task Load Index): Results of empirical and theoretical research, Advances in Psychology 52 (1988) 139-183.

[8] J.T. Kajiya, The rendering equation, Computer Graphics (Proc. SIGGRAPH'86) 20(4) (1986) 143150.

[9] M.H. Kalos, On the Estimation of Flux at a Point by Monte Carlo, Nuclear Science and Engineering, 16(1) (1963) 111-117.

[10] A. Keller, Instant radiosity, in: SIGGRAPH '97 Proceedings of the 24th annual conference on Computer graphics and interactive techniques, 1997, pp.49-56.

[11] C. Klein-Braley, U. Raatz, A survey of research on the C-Test, Language Testing 1 (1984) 134146.

[12] C. Moosmann, C. Vandahl, LiTG-Fragebogen zur Bewertung von Lichtsituationen. Proceedings of the Lux Junior, Dörnfeld, Germany, 2015.

[13] G.H. Scheir, et al, Calculation of the Unified Glare Rating based on luminance maps for uniform and non-uniform light sources, Building and Environment 84 (2015) 60-67. 